\title{
FSR 1716: A New Milky Way Globular Cluster Confirmed Using VVV RR Lyrae Stars
}

\author{
Dante Minniti ${ }^{1,2,3}$, Tali Palma ${ }^{2,1}$, Istvan Dékány ${ }^{4}$, Maren Hempel ${ }^{5}$, Marina Rejkuba ${ }^{6,7}$, Joyce Pullen ${ }^{2}$, Javier Alonso-García ${ }^{8,2}$,
} Rodolfo Barbá9 ${ }^{9}$, Beatriz Barbuy ${ }^{10}$, Eduardo Bica ${ }^{11}$, Charles Bonatto ${ }^{11}$, Jura Borissova ${ }^{12,2}$, Marcio Catelan ${ }^{5,2}$, Julio A. Carballo-Bello ${ }^{5,2}$, Andre Nicolas Chene ${ }^{13}$, Juan José Clariá ${ }^{14}$, Roger E. Cohen ${ }^{15}$, Rodrigo Contreras Ramos ${ }^{5,2}$, Bruno Dias $^{16}$, Jim Emerson ${ }^{17}$, Dirk Froebrich ${ }^{18}$, Anne S. M. Buckner ${ }^{18,19}$, Douglas Geisler ${ }^{15}$, Oscar A. Gonzalez ${ }^{20}$, Felipe Gran ${ }^{5}$, Gergely Hagdu ${ }^{5}$, Mike Irwin ${ }^{21}$, Valentin D. Ivanov ${ }^{6,16}$, Radostin Kurtev ${ }^{12,2}$, Philip W. Lucas ${ }^{22}$, Daniel Majaess ${ }^{23,24}$, Francesco Mauro $^{15}$, Christian Moni-Bidin ${ }^{25}$, Camila Navarrete ${ }^{5}$, Sebastian Ramírez Alegría ${ }^{12,2}$, Roberto K. Saito ${ }^{26}$, Elena Valenti ${ }^{6}$, and Manuela Zoccali ${ }^{5,2}$

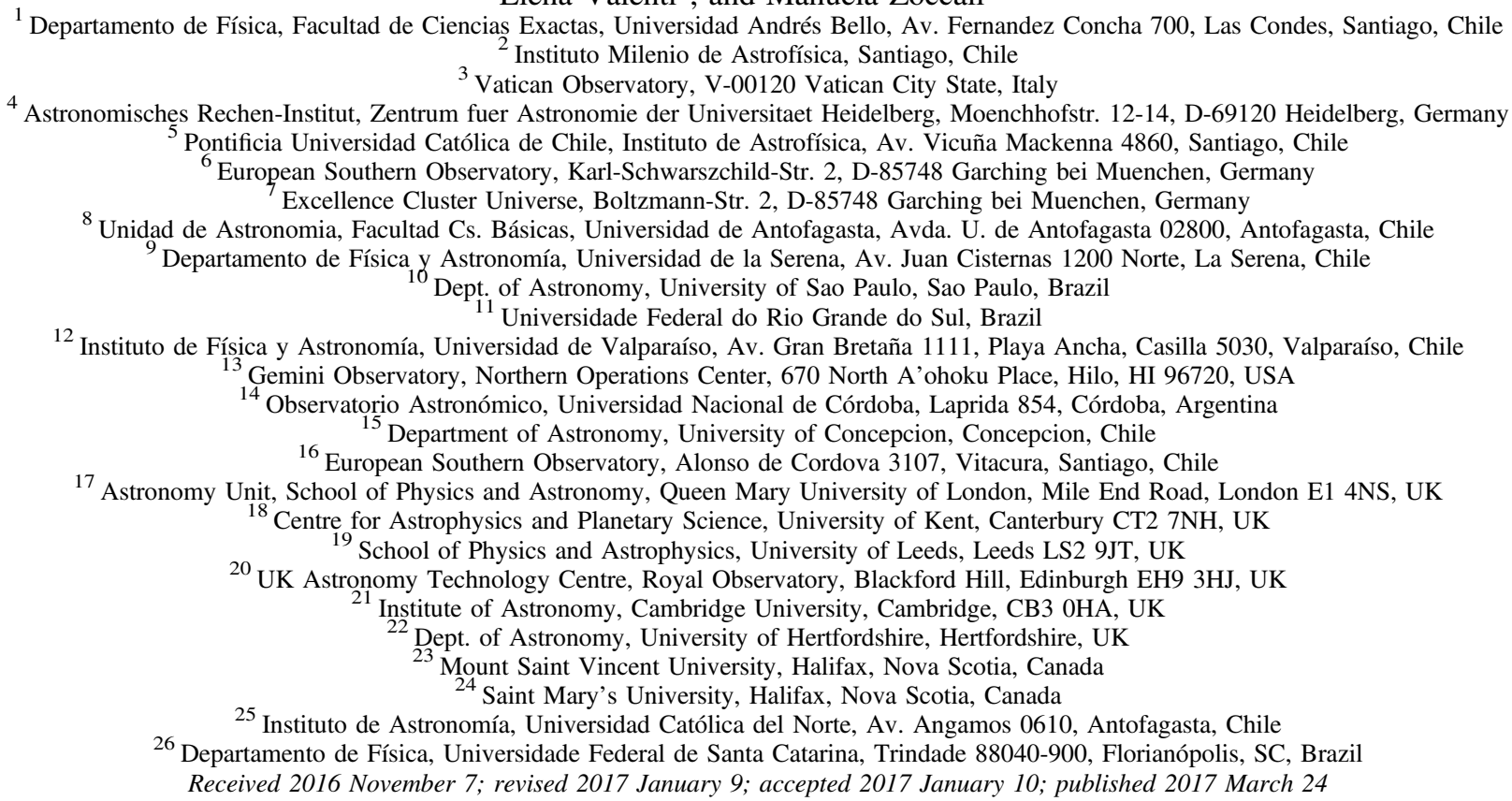

\begin{abstract}
We use deep multi-epoch near-IR images of the VISTA Variables in the Vía Láctea (VVV) Survey to search for RR Lyrae stars toward the Southern Galactic plane. Here, we report the discovery of a group of RR Lyrae stars close together in VVV tile d025. Inspection of the VVV images and PSF photometry reveals that most of these stars are likely to belong to a globular cluster that matches the position of the previously known star cluster FSR 1716. The stellar density map of the field yields a $>100 \sigma$ detection for this candidate globular cluster that is centered at equatorial coordinates R.A. ${ }_{J 2000}=16: 10: 30.0$, decl. ${ }_{J 2000}=-53: 44: 56$ and galactic coordinates $l=329.77812, b=-1.59227$. The color-magnitude diagram of this object reveals a well-populated red giant branch, with a prominent red clump at $K_{s}=13.35 \pm 0.05$, and $J-K_{s}=1.30 \pm 0.05$. We present the cluster RR Lyrae positions, magnitudes, colors, periods, and amplitudes. The presence of RR Lyrae indicates an old globular cluster, with an age $>10$ Gyr. We classify this object as an Oosterhoff type I globular cluster, based on the mean period of its RR Lyrae type ab, $\langle P\rangle=0.540$ days, and argue that this is a relatively metal-poor cluster with $[\mathrm{Fe} / \mathrm{H}]=-1.5 \pm 0.4 \mathrm{dex}$. The mean extinction and reddening for this cluster are $A_{K_{s}}=0.38 \pm 0.02$ and $E\left(J-K_{s}\right)=0.72 \pm 0.02 \mathrm{mag}$, respectively, as measured from the RR Lyrae colors and the near-IR colormagnitude diagram. We also measure the cluster distance using the RR Lyrae type ab stars. The cluster mean distance modulus is $(m-M)_{0}=14.38 \pm 0.03 \mathrm{mag}$, implying a distance $D=7.5 \pm 0.2 \mathrm{kpc}$ and a Galactocentric distance $R_{G}=4.3 \mathrm{kpc}$.
\end{abstract}

Key words: globular clusters: general - galaxies: photometry - stars: variables: RR Lyrae

\section{Introduction}

RR Lyrae variable stars are distance indicators that can be used to detect substructures in the Milky Way halo (Baker \& Willman 2015). Indeed, they have been recently used to find streams far out in the Milky Way halo (e.g., Ivezić et al. 2004; Keller et al. 2008; Sesar et al. 2010; Drake et al. 2014; Duffau et al. 2014; Munari et al. 2014; Torrealba et al. 2015) avoiding the Galactic plane regions. Because of high extinction and stellar crowding, many globular clusters may remain undetected toward the Galactic plane (Ivanov et al. 2005). The VISTA Variables in the Vía Láctea (VVV) Survey could detect some of them as well as measuring their astrophysical parameters in the near-IR (Minniti et al. 2010). Our previous cluster searches were based on the identification (visually or 
automatically) of field stellar overdensities, successfully yielding new open and globular clusters (Borissova et al. 2011, 2014; Minniti et al. 2011; Moni Bidin et al. 2011; Barbá et al. 2015). Our new globular cluster search concentrated in the Galactic plane. The places where suspected incompleteness and where the last few globular clusters were found are deep in the bulge or far out in the halo (e.g., Ivanov et al. 2005; Baker \& Willman 2015). Applying the idea of Baker \& Willman (2015) to the inner Galaxy and approaching it in a complementary way, we use the RR Lyrae stars as tracers to mark the location of old and metal-poor globular clusters hidden behind regions of large extinction in the Galactic plane. Indeed, the present globular cluster search found a new globular cluster embedded in the middle of the disk. Our discovery underscores the need to search also the Milky Way disk for missing globular clusters.

We have used the VVV Survey data to search for RR Lyrae type $a b$ (hereafter RRab) in extremely reddened environments of the Milky Way, including the Galactic disk, bulge, and center (e.g., Dékány et al. 2013; Gran et al. 2016; Minniti et al. $2016,2017)$. We have found hundreds of RRab stars located in a thin strip across the Galactic disk (Minniti et al. 2017), at Galactic latitudes $-2.24<b<-1^{\circ} .05$, and Galactic longitudes $\left(295^{\circ}<l<350^{\circ}\right)$. These regions are very crowded in the near-IR, having very high and variable reddenings. The RRab stars, however, are excellent reddening and distance indicators. Because they lie in the narrow instability strip region of the color-magnitude diagram, their intrinsic colors are well known and show a very narrow spread in the near-IR. For example, the RRab of the globular cluster $\omega$ Cen have $0.22<\left(J-K_{s}\right)<0.35$ (Navarrete et al. 2015). Therefore, it can be assumed an intrinsic (unreddened) color $\left(J-K_{s}\right)_{0}=0.21 \pm 0.05$ for any individual (unblended) RR Lyrae. The reddenings in the Galactic disk fields explored here range from $E\left(J-K_{s}\right)=0.2$ to $3.1 \mathrm{mag}$, and therefore such uncertainty in the intrinsic RRab colors $(\sigma=0.05 \mathrm{mag})$ is comparatively negligible.

We then searched our RR Lyrae database with these ideas in mind. Indeed, the RR Lyrae maps of the Galactic disk showed overdensities, some of which can be just random groupings. However, we found that a few of these groups are real (Minniti et al. 2017). For example, there are five RR Lyrae stars located at the same distance centered in the VVV tile d031 at $l=339.2$, $b=-1$.8. These RR Lyrae stars turned out to be members of the known globular cluster FSR 1735 (Carballo-Bello et al. 2016). In this Letter, we report the discovery of another group of RR Lyrae stars, all of them located at the same distance in a field centered in the VVV tile d025 at $l=329.8, b=-1^{\circ} .6$. We find a star cluster in our images (that we initially called VVVGC005), and that turned out to be very close to the previously known cluster FSR 1716 (Froebrich et al. 2007).

\section{RR Lyrae Selection}

The search for RR Lyrae type ab in the disk of the Milky Way (Minniti et al. 2017) revealed a few groups of RR Lyrae type ab located at the same distance in the same fields. The group considered here (listed in Table 1) is one of the largest overdensities outside the bulge, consisting of about a dozen RR Lyrae stars, located in a small region near the edge of the VVV tile d025, at $l=329.8, b=-1.6$. Most of them are located at about the same distance, while there are a few other candidates that appear to be foreground or background RR Lyrae stars. The selection of candidate cluster members was restricted to objects within $\sim 15$ arcmin of the cluster center, with eight of them (five RRab plus three RRc) being most likely cluster members because they are more tightly packed at the position of the cluster. The remaining RR Lyrae are more distant in the sky, and proper motions are needed in order to establish cluster membership. In addition, there are a few other candidate RR Lyrae variable stars in the field, for which additional epochs of observation are needed in order to confirm them as bona fide RR Lyrae stars.

We have initially concentrated on the search for fundamental mode pulsators (RR Lyrae type ab stars) that have asymmetric light curves in order to avoid contamination from eclipsing binaries (Minniti et al. 2017). Further inspection of the VVV light curves revealed four candidate RR Lyrae type $\mathrm{c}$ in this region. Although these are also listed in Table 1, they were not used to determine the cluster parameters (reddening, distance, metallicity) because of the possibility of contamination for eclipsing binaries. Table 1 lists the Galactic $(l, b)$ coordinates, $K_{s}$-band amplitudes, periods (in days), mean near-IR magnitudes and colors, and types for the RR Lyrae sample. Figure 1 shows the light curves of the RR Lyrae that are well classified (listed without a question mark in Table 1).

\section{VVV Images and Color-Magnitude Diagrams}

A close inspection of the deep VVV images of tile d025 where the RR Lyrae group is located clearly reveals a bona fide star cluster that we initially called VVV-GC05 (Figure 2). This turns out to be close to the position of the previously known cluster FSR 1716 (Froebrich et al. 2007). This cluster can also be seen in the GLIMPSE infrared images (Benjamin et al. 2005). The cluster FSR 1716 was classified as an "open-globular cluster" on the basis of NTT photometry (Froebrich et al. 2008). This cluster was also listed by Kharchenko et al. (2013) and Buckner \& Froebrich (2013, 2014), arguing for an open cluster nature as well, but not much more is known about this object. Bonatto \& Bica (2008) studied the color-magnitude diagram (CMD) from 2MASS and argued that FSR 1716 is an old open cluster $(\sim 7$ Gyr) at a distance of $D=0.8 \pm 0.1 \mathrm{kpc}$, without discarding the possibility of its being a loose globular cluster. They argue that this cluster is similar to the old open cluster NGC 188. They also examined the alternative that, if this a $12 \mathrm{Gyr}$ old globular cluster, its distance would be $D=2.3 \pm 0.3 \mathrm{kpc}$ for $A v=6.3 \pm 0.4$.

We find that the cluster in the VVV images is centered about 100 arcsec away from the original position of the cluster FSR 1716 from Froebrich et al. (2007), but we argue that they are the same object, and we will use this name hereafter. Figure 3 shows a 2D density histogram, with a clear maximum of stars at the position of this object. A Koposov test (Koposov et al. 2007, 2008) reveals that the cluster is centered at R.A. $J_{2000}=16: 10: 30.0$, decl. ${ }_{J 2000}=-53: 44: 56$ and Galactic coordinates $l=329.77812, b=-1.59227$, which are the final coordinates we adopted here. There is a bright saturated foreground star at the western edge of the cluster, and even though it might affect the measured shape, this does not affect the coordinate determination. In spite of contamination by other bright red stars in the field (Figure 3), we measured that this cluster has an elliptical shape $(b / a \sim 0.7)$ and a total extension 
Table 1

Photometric Observations of RR Lyrae in the Field of FSR 1716

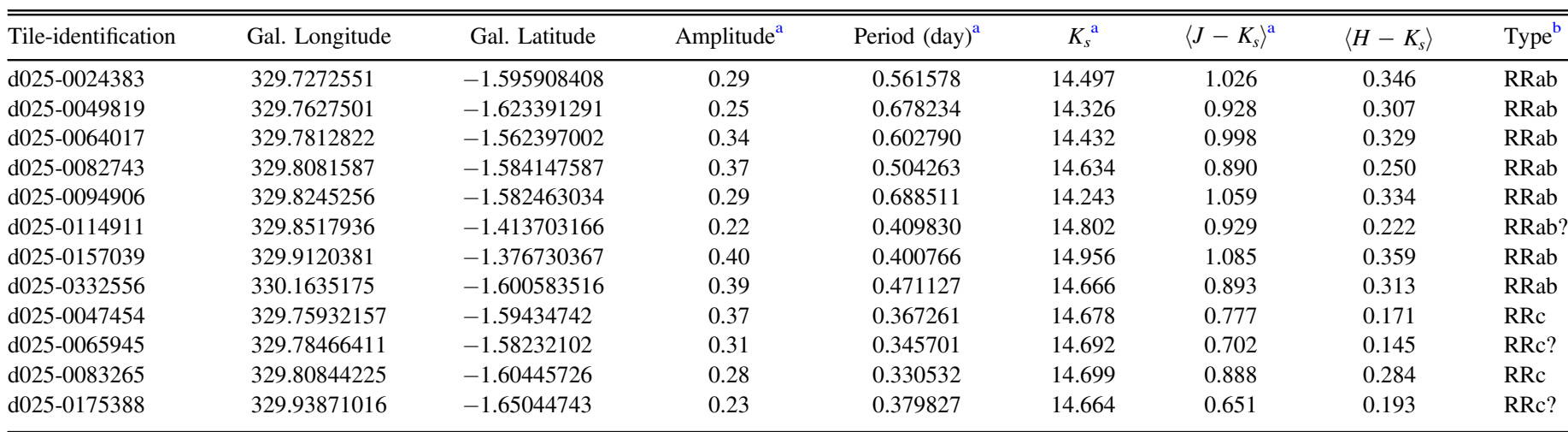

Notes.

${ }^{\text {a }}$ Typical photometric errors are $\sigma_{K_{s}}=0.01 \mathrm{mag}$ and $\sigma_{J, H}=0.03$ mag. Periods are accurate to $10^{-5}$ days, and $K_{s}$-band amplitude errors are of the order of $\sigma_{A}=0.02 \mathrm{mag}$

${ }^{\mathrm{b}}$ Variables with uncertain classification are labeled with a question mark, and their light curves are omitted from Figure 1.
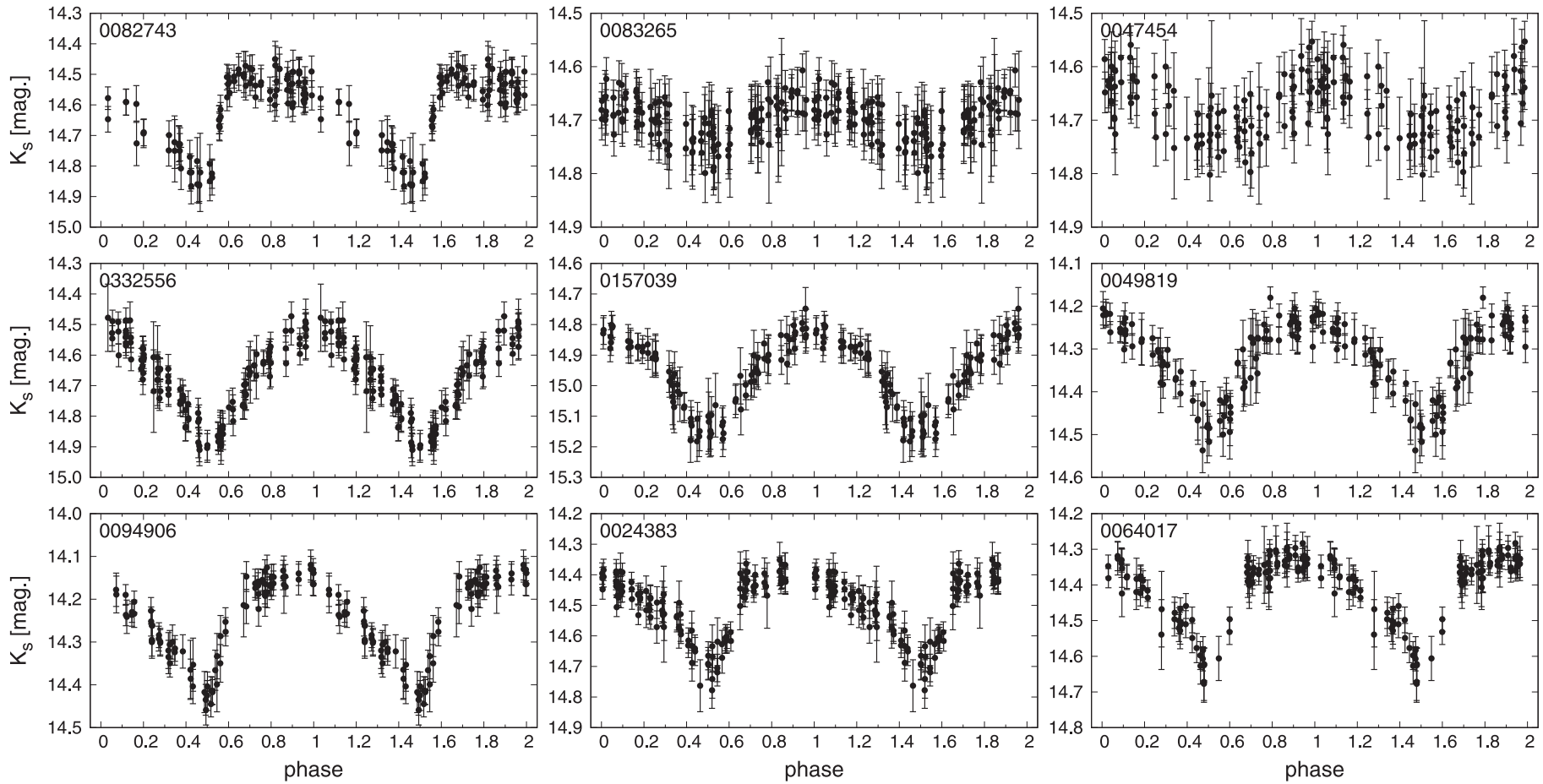

Figure 1. Phased light curves for the candidate RR Lyrae type ab of the new globular cluster FSR 1716 that are classified as certain RRab or RRc. The more dubious variables listed with a question mark in Table 1 are not shown.

of $a \sim 500$ pix ( $\sim 3$ arcmin in the near-IR). This is equivalent to a radius of $\sim 3.5 \mathrm{pc}$ at a distance of $D=8 \mathrm{kpc}$, consistent with the sizes of known globular clusters.

The CMD centered on the new globular cluster (Figure 4) is different from that of the surrounding region. This CMD reveals a populated red giant branch (RGB), with a prominent red clump (core-He burning stars). Because the field is very crowded and with variable reddening, we decontaminate the cluster CMD following the procedures adapted for the VVV images as described by Palma et al. (2016). Briefly, we took a small region with radius 3.0 arcmin centered on FSR 1716, and four equivalent background area regions located 15 arcmin away from the cluster. We tried a few background areas that exhibited similar apparent reddenings. The decontamination was done by eliminating the stars in the cluster CMD that appear as the closest neighbors in the background CMDs. After a few iterations, we built the decontaminated cluster CMD shown in the third panel of Figure 4. This CMD clearly exhibits the cluster RGB and red clump at $K_{s}=13.35 \pm 0.05$ and $J-K_{s}=1.30 \pm 0.05$. The luminosity function (rightmost panel of Figure 4) also shows the red clump. However, still some contaminating stars belonging to the Galactic disk remain (blue stars located in the left region of the CMD with $J-K_{s}<0.8 \mathrm{mag}$ ), and proper motions are clearly needed in order to better clean up the CMD of this cluster, especially in the turnoff region that is close to our photometric limit. 


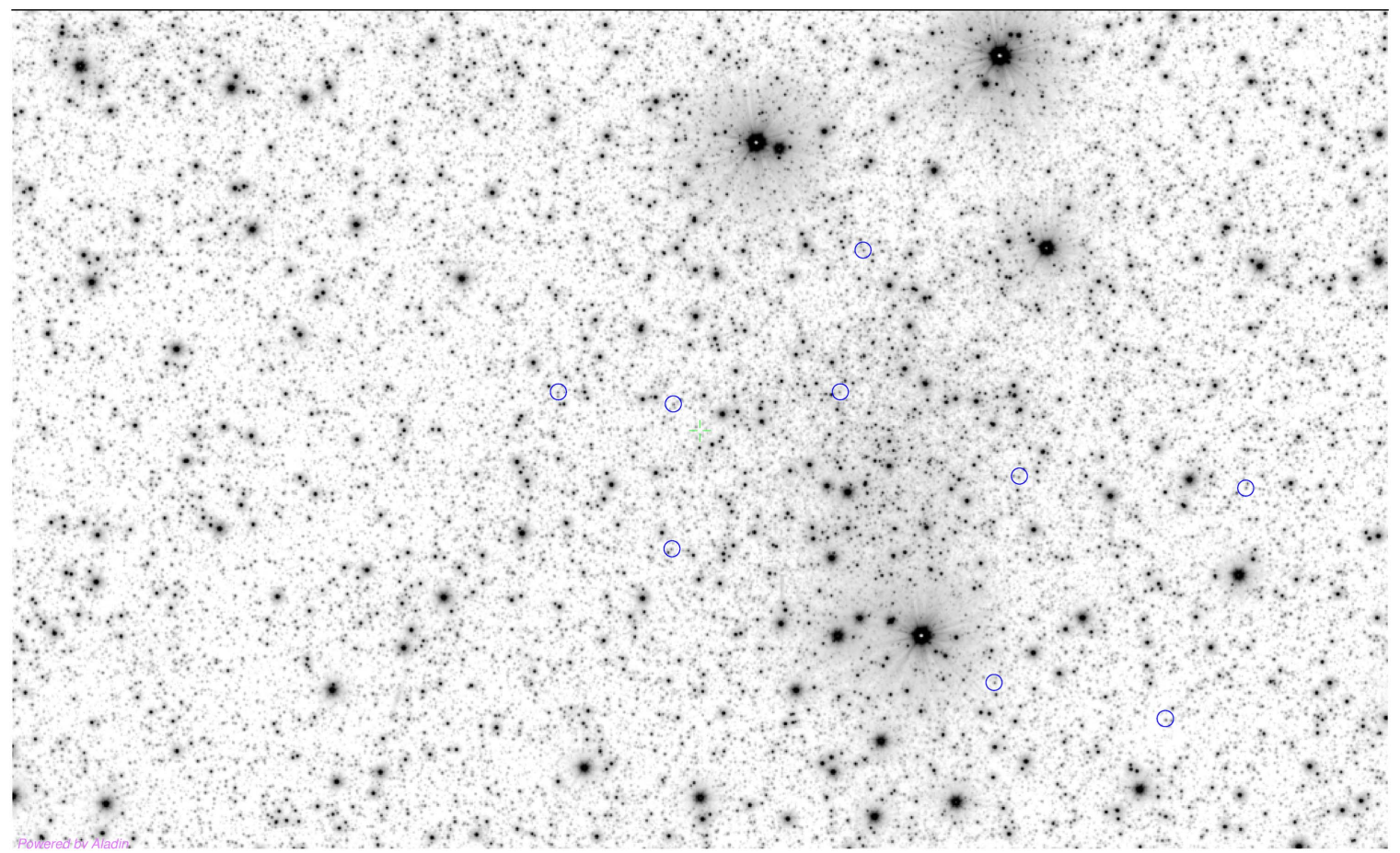

Figure 2. Illustration of the cluster stellar field. Deep Ks-band image of the field of the new globular cluster FSR 1716 located at the far right in order to illustrate the field density. This image covers $13 \times 7 \operatorname{arcmin}^{2}$ of VVV tile d025 and is oriented along Galactic coordinates $l, b$.
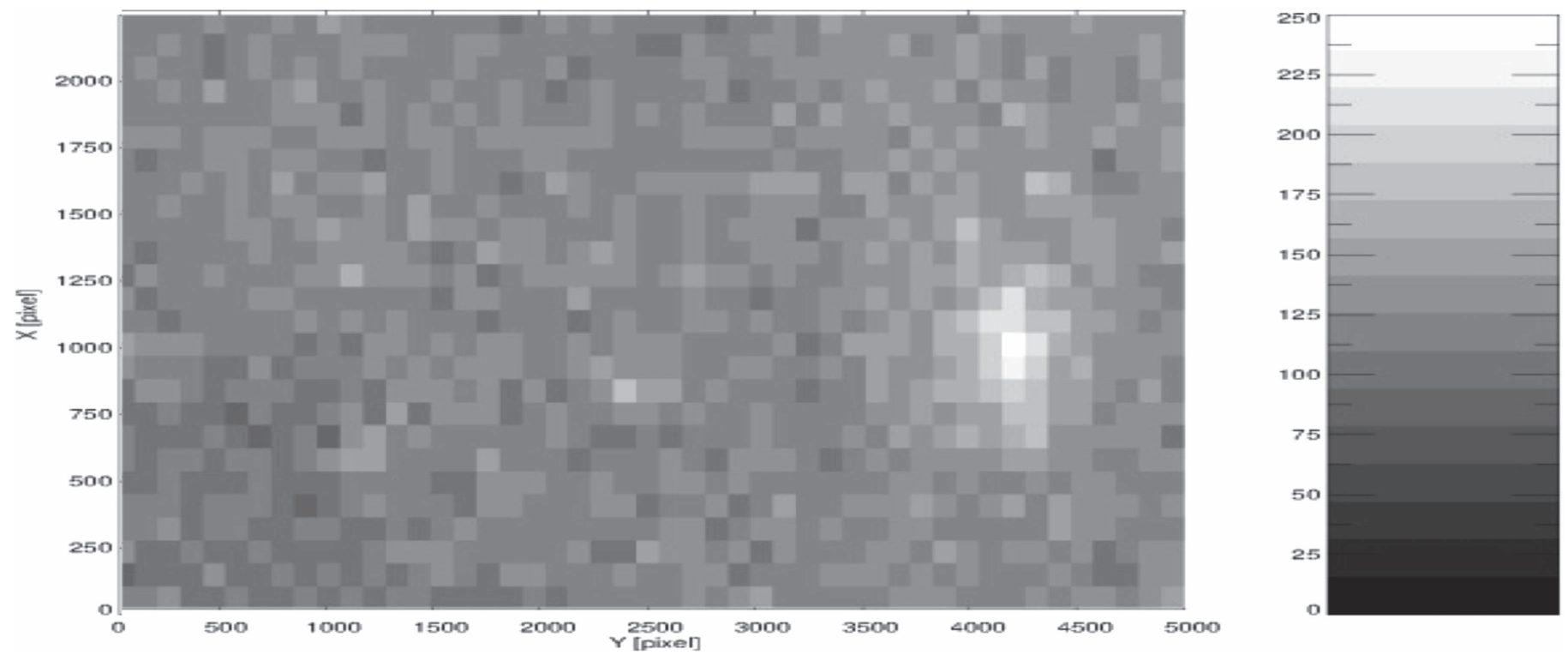

Figure 3. Density map of the VVV-GC005 surrounding region. There is a clear maximum of stars at pixels (4200, 1000), marking the position of FSR 1716 (white area). The significance scale on the right illustrates that the cluster's significance above the background is $>100$.

\section{Reddening and Extinction}

The reddening toward low latitude fields in the Galactic plane is large and non-uniform. There are previous estimates for the reddening in the field of FSR 1716, which show a significant spread, and since there was no general agreement, the field extinction still was uncertain. Froebrich et al. (2008) obtained a reddening value $E\left(J-K_{s}\right)=0.57$ (equivalent to $A_{K_{s}}=0.30$ ), based on 2MASS near-IR photometry. Bonatto \& Bica (2009) derived an extinction value $A_{V}=6.3 \pm 0.2$ (equivalent to $A_{K_{s}}=0.7$ ), also based on 2MASS near-IR photometry. Besides, the maps of Schlafly \& Finkbeiner (2011) and Schlegel et al. (1998) give $A_{K_{s}}=0.86$, and $1.01 \mathrm{mag}$, respectively (in the UKIRT system which should be similar to the VISTA $K_{s}$ system), for this region of tile $\mathrm{d} 025$. These extinctions are equivalent to $A_{V}=7.8$ and $9.1 \mathrm{mag}$, respectively, showing that the field is indeed very reddened.

The reddening determination for FSR 1716 is very important as it lies in the Galactic plane and the extinction value impacts on the distance determination. Fortunately, the RR Lyrae stars are excellent reddening indicators, as are the clump giants. We can estimate the mean reddening and extinction of the globular cluster FSR 1716 using the photometry of the five RR Lyrae type ab listed in Table 1 that are closest to the cluster center. 


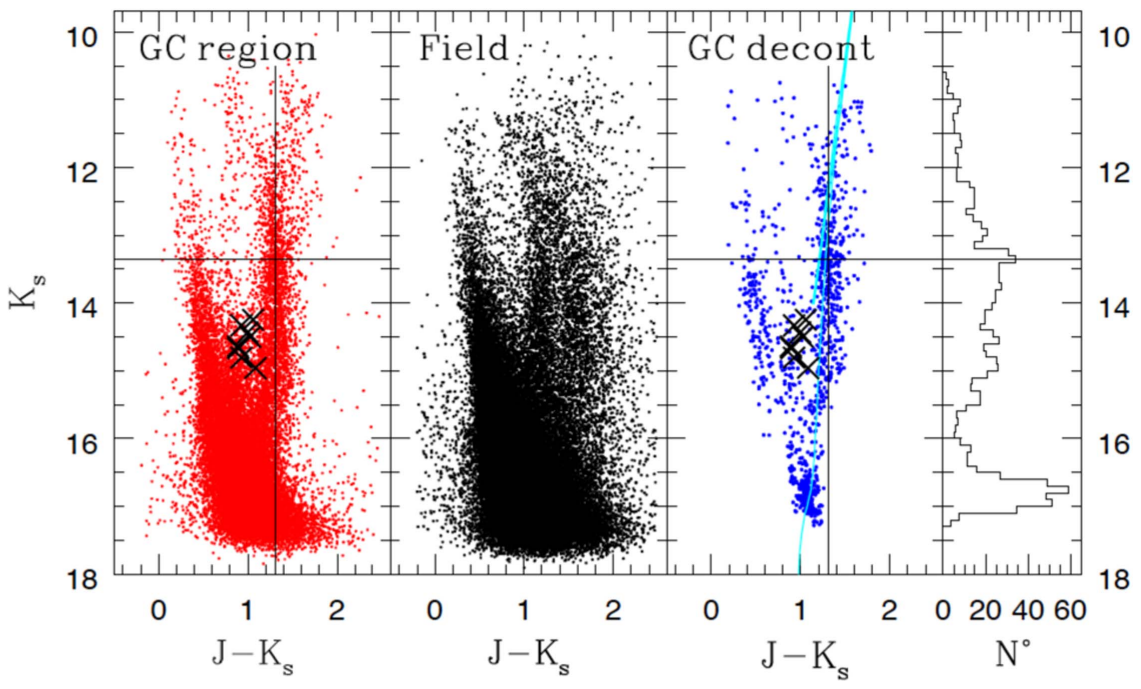

Figure 4. First panel: VVV PSF near-IR CMD for a 3 arcmin field centered on FSR 1716. The position of the globular cluster red clump is marked at $K_{s}=13.35$ and $J-K_{s}=1.31$. The location of the globular cluster RR Lyrae type ab found here is indicated with large black crosses. Second panel: CMD of the surrounding comparison field. The main sequence of the Galactic disk is seen at $J-K_{s} \sim 0.5$. Third panel: decontaminated VVV PSF near-IR CMD for VVV-GC005. The points are the globular cluster stars within 3 arcmin of the cluster center, while the number of field stars has been minimized by the statistical decontamination procedure. The globular cluster RGB is well populated and the red clump is well defined. The main-sequence turnoff is located just below the faintest magnitudes. A 10 Gyr isochrone for $[\mathrm{Fe} / \mathrm{H}]=-1.3$ dex from Bessan et al. (2012) is plotted for comparison. The points with $J-K_{s}<0.8$ are the remaining main sequence of a foreground Galactic disk young population along the line of sight to the cluster. Fourth panel: luminosity function for the decontaminated globular cluster, clearly showing the location of the red clump.

The observed mean color of these cluster RR Lyrae type ab is $J-K_{s}=0.95 \pm 0.05$. The mean intrinsic (unreddened) color of RR Lyrae type ab should be $J-K_{s}=0.21 \pm 0.05$, from which we derive a cluster reddening value of $E\left(J-K_{s}\right)=0.74 \pm 0.07$ mag. This reddening corresponds to $A_{K_{s}}=0.39$, using the extinction ratio $A_{K_{s}} / E\left(J-K_{s}\right)=0.528$ of Nishiyama et al. (2009), adopted for this work. The choice of a different extinction ratio gives a measure of the external uncertainty that will be used in estimating the distance uncertainties in Section 5. For example, Cardelli et al. (1989) give $A_{K_{s}} / E\left(J-K_{s}\right)=0.72$, and AlonsoGarcía et al. (2015) give $A_{K_{s}} / E\left(J-K_{s}\right)=0.44 \pm 0.03$, yielding $A_{K_{s}}=0.53$ and 0.33 , respectively.

We also used for this field the reddening maps of M. Irwin et al. (2016, private communication) to obtain $E$ $\left(J-K_{s}\right)=0.75$ and $A_{K s}=0.40$. These maps for the VVV disk fields were made using the field red clump stars, following the procedure of Gonzalez et al. (2012). Considering the wide range of values published in the literature, this value is consistent with the previous determinations.

In order to obtain a reddening based on the red clump, we adopted a red clump mean intrinsic color $\left(J-K_{s}\right)_{0}=$ $0.61 \pm 0.01$, following Alves et al. (2002), Pietrzynski et al. (2003), Grocholski \& Sarajedini (2002), and Minniti et al. (2011). The observed mean red clump color is $J-K_{s}=1.32 \pm 0.05$ (Figure 4), yielding $E\left(J-K_{s}\right)=0.71$, and $A_{K_{s}}=0.38 \mathrm{mag}$, in excellent agreement with the RR Lyrae determination.

We adopted the mean reddening and extinction values $E\left(J-K_{s}\right)=0.72$ and $A_{K_{s}}=0.38 \mathrm{mag}$, determined from the position of the red clump in the CMD and from the RR Lyrae type ab stars. This is equivalent to $A_{V}=3.5 \mathrm{mag}$, half the value from Schlegel et al. (1998), Bica (2008), and Schlafly \& Finkbeiner (2011).

\section{Distance, Metallicity, and Age}

We can perform two independent distance measurements for this new globular cluster using: (i) RR Lyrae stars and (ii) the clump giants, expecting the first method to be more accurate. We used the period-luminosity relation for Galactic RR Lyrae type ab: $M_{K_{s}}=-2.53 \times \log (P)-0.95$ from Muraveva et al. (2015) to compute individual distances. Adopting $A_{K_{s}}=0.39$, the mean distance modulus for the 8 RRab from Table 1 is $(m-M)_{0}=14.40$, equivalent to $D=7.6 \pm 0.3 \mathrm{kpc}$, where the error is the sigma of the distribution from the dispersion about the period-luminosity relation. However, there are 3 $\mathrm{RRab}$ that are more distant from the cluster (d025-0114911, d025-0157039, and d025-0332556), and since we do not know the cluster tidal radius, their membership is insecure. Nonetheless, restricting the computation to the 5 RRab located closer to the cluster center (considered to be the best cluster members) yields a very similar result: $D=7.5 \pm 0.2 \mathrm{kpc}$.

In order to obtain a distance based on the cluster red clump, we adopted a red clump mean intrinsic magnitude $M_{K_{s}}=-1.65 \pm 0.03$ and $J-K_{s}=0.61 \pm 0.01$, following Alves et al. (2002), Pietrzynski et al. (2003), Grocholski \& Sarajedini (2002), and Minniti et al. (2011). Figure 4 shows that the globular cluster RGB is well defined. We are able to measure the location of the red clump from the statistically decontaminated CMD of Figure 4 at $K_{s}=13.35 \pm 0.05$ and $J-K_{s}=1.31 \pm 0.05$. The red clump gives $E\left(J-K_{s}\right)=0.70$ and $A_{K_{s}}=0.38 \mathrm{mag}$ from above. This extinction yields a clump giant distance modulus $(m-M)_{0}=14.62$, equivalent to a distance of $D=8.4 \pm 0.3 \mathrm{kpc}$.

Alternatively, assuming $A_{K_{s}}=0.40$ (from the reddening maps), the distance modulus should be $(m-M)_{0}=14.60$. Froebrich et al. (2008) found two peaks in the $K$-band luminosity function, one at $K=13.1$ and another at $K=13.7$. On this basis, they argue that the cluster can be at $D=7 \mathrm{kpc}$ for 
an age of $\sim 2 \mathrm{Gyr}$, but it could also be as close to $D=5 \mathrm{kpc}$, if the age is $>10 \mathrm{Gyr}$ (for their adopted extinction $A_{K}=0.57$ ).

The distance difference measured using these two population tracers is significant $(0.8 \mathrm{kpc})$, but still within the errors given that the red clump distance is more uncertain. This is a cluster embedded in the Galactic plane, and from this discussion, it is clear that reddening is a critical parameter for its distance determination. We adopted a final a mean distance, $D=7.5 \pm$ $0.2 \mathrm{kpc}$, from the more accurate RR Lyrae determination. The corresponding Galactocentric distance is $R_{G}=4.3 \mathrm{kpc}$.

In the absence of spectroscopic data, we can estimate the cluster photometric metallicity based on the RR Lyrae and RGB properties. Based on the mean period of its RR Lyrae type $\mathrm{ab},\langle P\rangle=0.607$ days, we classified FSR 1716 as an Oosterhoff type I globular cluster (even though it is at the edge of the Oosterhoff intermediate clusters; Catelan 2004). We applied the period-amplitude-metallicity relations of Alcock et al. (2000), Yang et al. (2010), and Feast et al. (2010) to find a mean metallicity $[\mathrm{Fe} / \mathrm{H}]=-1.5 \pm 0.3 \mathrm{dex}$, based on the 5 RR Lyrae type ab stars closest to the center (including the more distant candidates d025-0114911, d025-0157039, and d0250332556 yields $[\mathrm{Fe} / \mathrm{H}]=-1.3 \pm 0.5 \mathrm{dex})$. The candidate $\mathrm{RRc}$ variables also point to the Oosterhoff type I nature (there are fewer RRc than RRab stars, and their periods are typical of RRc of Oosterhoff type I clusters).

Valenti et al. (2004), Sollima et al. (2004), and Cohen et al. (2016) presented the fiducial RGBs for globular clusters in the $K_{s}$ versus $J-K_{s}$ plane. It is possible to measure the photometric indices along the RGB in the $\left[M_{K_{s}},\left(J-K_{s}\right)_{0}\right]$ absolute plane, namely, the magnitude at fixed color, the colors at fixed magnitudes, and the slope of the RGB. A comparison of the unreddened RGB of FSR 1716 with these fiducial globular cluster RGBs reveals that our cluster should be relatively metalpoor. Adopting a mean reddening $E\left(J-K_{s}\right)=0.72$, the RGB fits well the fiducial sequences for the globular clusters $\omega$ Cen at $[\mathrm{Fe} / \mathrm{H}] \sim-1.5$ dex (Navarrete et al. 2015), and Terzan 1 at $[\mathrm{Fe} / \mathrm{H}] \sim-1.3$ dex (Valenti et al. 2010). However, the RGB color spread is significant, indicating the presence of differential reddening in the field. Then, we cannot discard good fits for RGBs over a wide range in metallicities, from about $[\mathrm{Fe} / \mathrm{H}]=-2.0$ (like the globular cluster M55) to $-0.7 \mathrm{dex}$ (like the globular cluster $47 \mathrm{Tuc}$ ). In summary, based on the RR Lyrae stars and on the RGB, we estimated that FSR 1716 is a metal-poor globular cluster with $[\mathrm{Fe} / \mathrm{H}]=-1.5 \pm 0.4$ dex. This metallicity should be considered uncertain so spectroscopic measurements are needed. These observations should be made with large telescopes because the brightest cluster member giants should reach $V \sim 18$ mag.

The cluster age would have been difficult to constrain, if it had not contained RR Lyrae stars. The presence of these variables indicates that this is an old ( $>10 \mathrm{Gyr})$ globular cluster, ruling out a young or intermediate-age star cluster. As mentioned before, the CMD is well fit by the fiducial line of the globular cluster $\omega$ Cen. We have also fitted $10 \mathrm{Gyr}$ old isochrones from Bressan et al. (2012) for the appropriate metallicity (see Figure 4). However, the globular cluster turnoff region is just beyond the limit of the photometry, and the isochrones can only be used to rule out younger ages.

\section{Conclusions}

We have discovered a compact group of RR Lyrae type ab stars toward the Milky Way southern plane, located at a common distance in the direction of VVV tile d025. These stars are centered at $l=329.77812, \quad b=-1.59227 ; \quad$ R.A. $J 2000=16: 10: 30.0$, decl. $J 2000=-53: 44: 56$, and appear to belong to the cluster FSR 1716, which is therefore identified as a new Galactic globular cluster. The CMD is consistent with that of a typical globular cluster, and the red clump is clearly seen in the cluster RGB at $K_{s}=13.35 \pm 0.05$ and $J-K_{s}=1.31 \pm 0.05$. We found eight type $\mathrm{ab}$ and four type c RR Lyrae in total within the field of this new globular cluster. We present accurate positions, near-IR magnitudes, colors, periods, and amplitudes for these stars. The high quality of the VVV Survey near-IR photometry allows us to measure the parameters for this cluster, like reddening, distance, metallicity, and age.

We estimated the mean reddening $\left(E\left(J-K_{s}\right)=0.74\right.$ mag $)$ and distance $(D=7.5 \pm 0.2 \mathrm{kpc})$ to the globular cluster FSR 1716 using the five RR Lyrae type ab candidate members that are more centrally concentrated and are therefore most likely cluster members. Judging by the presence of RR Lyrae variable stars, this cluster seems to be an old ( $>10 \mathrm{Gyr})$, globular cluster of Oosterhoff type I. Its metallicity, estimated based using both RR Lyrae type ab stars and the RGB color, is $[\mathrm{Fe} / \mathrm{H}]=-1.5 \pm 0.4$ dex.

We gratefully acknowledge data from the ESO Public Survey program ID 179.B-2002 taken with the VISTA telescope and products from the Cambridge Astronomical Survey Unit (CASU). Support is provided by the BASAL Center for Astrophysics and Associated Technologies (CATA) through grant PFB-06, and the Ministry for the Economy, Development and Tourism, Programa Iniciativa Científica Milenio grant IC120009, awarded to the Millennium Institute of Astrophysics (MAS). D.M., M.Z., C.M.B., and R.K. acknowledge support from FONDECYT Regular grants Nos. 1130196, 1150345, 1150060, and 1130140, respectively. We are grateful to the Aspen Center for Physics where our work was supported by National Science Foundation grant PHY1066293 and by a grant from the Simons Foundation (D.M. and M.Z.). J.A-G. acknowledges support from FONDECYT Iniciación grant 11150916. B.B. is partially supported by CNPq and Fapesp. G.H. is supported by CONICYT-PCHA (Doctorado Nacional 2014-63140099). R.E.C. acknowledges the Gemini-CONICYT for Project 32140007. A.C. is supported by Gemini Observatory, operated by AURA Inc., on behalf of the international Gemini partnership of Argentina, Brazil, Canada, Chile, and USA. R.K.S. acknowledges support from $\mathrm{CNPq} /$ Brazil through project 310636/2013-2. We acknowledge use of the SIMBAD database, operated at CDS, Strasbourg, France.

\section{References}

Alcock, C., Allsman, R. A., Alves, D. R., et al. 2000, AJ, 119, 2194 Alonso-García, J., Dekany, I., Catelan, M., et al. 2015, AJ, 149, 99 Alves, D., Rejkuba, M., Minniti, D., \& Cook, K. 2002, ApJL, 573, L51 Baker, M., \& Willman, B. 2015, AJ, 150, 160

Barbá, R. H., Roman-Lopes, A., Nilo Castellon, J. L., et al. 2015, A\&A, 581,120

Benjamin, R. A., Churchwell, E., Blaber, B. L., et al. 2005, ApJL, 630, L149 Bonatto, C., \& Bica, E. 2008, A\&A, 491, 767

Bonatto, C., \& Bica, E. 2009, MNRAS, 397, 1032

Borissova, J., Bonatto, C., Kurtev, R., et al. 2011, A\&A, 532, 131

Borissova, J., Chene, A.-N., Ramírez Alegría, S., et al. 2014, A\&A, 569, 24

Bressan, A., Marigo, P., Girardi, L., et al. 2012, MNRAS, 427, 127

Buckner, A., \& Froebrich, D. 2013, MNRAS, 436, 1465

Buckner, A., \& Froebrich, D. 2014, MNRAS, 444, 290 
Carballo-Bello, J., Ramirez Alegriia, S., Borissova, J., et al. 2016, MNRAS, 462,501

Cardelli, J. A., Clayton, G. C., \& Mathis, J. S. 1989, ApJ, 345, 245

Catelan, M. 2009, Ap\&SS, 320, 261

Cohen, R. E., Moni Bidin, C., Mauro, F., et al. 2017, MNRAS, 464, 1874

Dékány, I., Minniti, D., Catelan, M., et al. 2013, ApJ, 776, 19

Drake, A., Graham, M. J., Djorgovski, S. G., et al. 2014, ApJS, 213, 9

Duffau, S., Vivas, A. K., Zinn, R., et al. 2014, A\&A, 566, 118

Feast, M. W., Abedigamba, O. P., \& Whitelock, P. A. 2010, MNRAS, 408, L76

Froebrich, D., Meusinger, H., \& Scholz, A. 2007, MNRAS, 377, L54

Froebrich, D., Meusinger, H., \& Scholz, A. 2008, MNRAS, 390, 1598

Gonzalez, O. A., Rejkuba, M., Zoccali, M., et al. 2012, A\&A, 543, 13

Gran, F., Minniti, D., Saito, R. K., et al. 2016, A\&A, 591, 145

Grocholski, A. J., \& Sarajedini, A. 2002, AJ, 123, 1603

Ivanov, V. D., Kurtev, R., \& Borissova, J. 2005, A\&A, 442, 195

Ivezić, Ž., Lupton, R., Schlegel, D., et al. 2004, in ASP Conf. Ser. 327, Satellites and Tidal Streams, ed. F. Prada, D. Martinez Delgado, \& T. J. Mahoney (San Francisco, CA: ASP), 104

Kharchenko, N. V., Piskunov, A. E., Schilbach, E., Röser, S., \& Scholz, R.-D. 2013, A\&A, 558, 53

Kharchenko, N. V., et al. 2013, A\&A, 558, 53

Kinman, T. D. 1959, MNRAS, 119, 134
Koposov, S., Belokurov, V., Evans, N. W., et al. 2008, ApJ, 686, 279

Koposov, S., de Jong, J. T. A., Belokurov, V., et al. 2007, ApJ, 669, 337

Minniti, D., Contreras-Ramos, R., Zoccali, M., et al. 2016, ApJL, 810, L20

Minniti, D., Dekany, I., Majaess, D., Palma, T., et al. 2017, AJ, in press

Minniti, D., Hempel, M., Toledo, I., et al. 2011, A\&A, 527, L81

Minniti, D., Lucas, P. W., Emerson, J. P., et al. 2010, NewA, 15, 433

Moni Bidin, C., Mauro, F., D. Geisler, D., et al. 2011, A\&A, 535, 33

Munari, U., Henden, A., \& Frigo, A. 2014, NewA, 21, 1

Muraveva, T., Palmer, M., Clementini, G., et al. 2015, ApJ, 807, 127

Navarrete, C., Contreras Ramos, R., Catelan, M., et al. 2015, A\&A, 577, 99

Nishiyama, S., Tamura, M., Hatano, H., et al. 2009, ApJ, 696, 1407

Palma, T., Minniti, D., Dekany, I., et al. 2016, NewA, 49, 50

Pietrzynski, G., Gieren, W., \& Udalski, A. 2003, AJ, 125, 2494

Schlafly, E. F., \& Finkbeiner, D. P. 2011, ApJ, 737, 103

Schlegel, D. J., Finkbeiner, D. P., \& Davis, M. 1998, ApJ, 500, 525

Sesar, V., Vivas, A. K., Duffau, S., \& Ivezic, Z. 2010, ApJ, 717, 133

Sollima, A., Ferraro, F. R., Origlia, L., et al. 2004, A\&A, 420, 173

Torrealba, G., Catelan, M., Drake, A. J., et al. 2015, MNRAS, 446, 2251

Valenti, E., Ferraro, F. R., \& Origlia, L. 2004, MNRAS, 351, 1204

Valenti, E., Ferraro, F. R., \& Origlia, L. 2010, MNRAS, 402, 1729

Yang, S. C., Sarajedini, A., Holzman, J. A., \& Garnett, D. R. 2010, ApJ, 724, 799 